\title{
VERY LOW MASS STELLAR AND SUBSTELLAR COMPANIONS TO SOLAR-LIKE STARS FROM MARVELS. IV. A CANDIDATE BROWN DWARF OR LOW-MASS STELLAR COMPANION TO HIP 67526
}

\author{
Peng Jiang ${ }^{1,2,24}$, Jian Ge ${ }^{1}$, Phillip Cargile ${ }^{3}$, Justin R. Crepp ${ }^{4}$, Nathan De LeE ${ }^{1,3}$, Gustavo F. Porto de Mello ${ }^{5,6}$, \\ Massimiliano Esposito $^{7,8}$, Letícia D. Ferreira ${ }^{5,6}$, Bruno Femenia $^{7,8}$, Scott W. Fleming ${ }^{1,9,10}$, B. Scott Gaudi ${ }^{11}$, \\ Luan Ghezzi ${ }^{6,12}$, Jonay I. GonZález Hernández ${ }^{7,8}$, Leslie HebB ${ }^{3}$, Brian L. LeE ${ }^{1,13}$, Bo MA ${ }^{1}$, Keivan G. Stassun ${ }^{3,14}$, \\ Ji WANG ${ }^{1}$, John P. Wisniewski ${ }^{15}$, ERic AgOl ${ }^{13}$, Dmitry Bizyaev ${ }^{16}$, Howard BreWington ${ }^{16}$, Liang Chang ${ }^{1}$, \\ Luiz Nicolaci da Costa ${ }^{6,12}$, Jason D. Eastman ${ }^{11,17,18}$, Garrett EbelKe ${ }^{16}$, Bruce Gary ${ }^{3}$, Stephen R. Kane ${ }^{19}$, Rui Li ${ }^{1}$, \\ Jian Liu ${ }^{1}$, Suvrath Mahadevan ${ }^{1,9,10}$, Marcio A. G. Maia ${ }^{6,12}$, Viktor Malanushenko ${ }^{16}$, Elena Malanushenko ${ }^{16}$, \\ Demitri Muna ${ }^{20}$, Duy Cuong Nguyen ${ }^{1}$, Ricardo L. C. Ogando ${ }^{6,12}$, Audrey Oravetz ${ }^{16}$, Daniel Oravetz ${ }^{16}$, Kaike Pan ${ }^{16}$, \\ Joshua Pepper ${ }^{3}$, Martin Paegert $^{3}$, Carlos Allende Prieto $^{7,8}$, Rafael Rebolo $^{7,21}$, Basilio X. Santiago ${ }^{6,22}$, \\ Donald P. Schneider ${ }^{9,10}$, Alaina C. Shelden Bradley ${ }^{16}$, Thirupathi Sivarani ${ }^{1,23}$, STePhanie Snedden $^{16}$, \\ J. C. van EyKen ${ }^{19}$, Xiaoke Wan ${ }^{1}$, Benjamin A. Weaver ${ }^{20}$, and Bo ZhaO ${ }^{1}$ \\ ${ }^{1}$ Astronomy Department, University of Florida, 211 Bryant Space Science Center, P.O. Box 112055, Gainesville, FL 32611, USA \\ ${ }^{2}$ Key Laboratory for Research in Galaxies and Cosmology, The University of Science and Technology of China, \\ Chinese Academy of Sciences, Hefei, Anhui 230026, China; jpaty@ mail.ustc.edu.cn \\ ${ }^{3}$ Department of Physics and Astronomy, Vanderbilt University, Nashville, TN 37235, USA \\ ${ }^{4}$ Department of Physics, University of Notre Dame, 225 Nieuwland Science Hall, Notre Dame, IN 46556, USA \\ ${ }^{5}$ Observatório do Valongo, Universidade Federal do Rio de Janeiro, Ladeira do Pedro Antônio, 43, CEP: 20080-090, Rio de Janeiro, RJ, Brazil \\ ${ }^{6}$ Laboratório Interinstitucional de e-Astronomia (LIneA), Rio de Janeiro, RJ 20921-400, Brazil \\ ${ }^{7}$ Instituto de Astrofísica de Canarias, C/Vía Láctea S/N, E-38200 La Laguna, Spain \\ ${ }^{8}$ Departamento de Astrofísica, Universidad de La Laguna, E-38205 La Laguna, Tenerife, Spain \\ ${ }^{9}$ Department of Astronomy and Astrophysics, The Pennsylvania State University, 525 Davey Laboratory, University Park, PA 16802, USA \\ ${ }^{10}$ Center for Exoplanets and Habitable Worlds, The Pennsylvania State University, University Park, PA 16802, USA \\ ${ }_{11}^{11}$ Department of Astronomy, The Ohio State University, 140 West 18th Avenue, Columbus, OH 43210, USA \\ 12 Observatório Nacional, Rua General José Cristino, 77, 20921-400 São Cristóvão, Rio de Janeiro, RJ, Brazil \\ ${ }^{13}$ Department of Astronomy, University of Washington, Box 351580, Seattle, WA 98195-1580, USA \\ ${ }^{14}$ Department of Physics, Fisk University, 1000 17th Ave. N., Nashville, TN 37208, USA \\ ${ }^{15}$ Homer L. Dodge Department of Physics and Astronomy, University of Oklahoma, 440 West Brooks Street, Norman, OK 73019, USA \\ ${ }^{16}$ Apache Point Observatory, P.O. Box 59, Sunspot, NM 88349-0059, USA \\ ${ }^{17}$ Las Cumbres Observatory Global Telescope Network, 6740 Cortona Drive, Suite 102, Santa Barbara, CA 93117, USA \\ ${ }^{18}$ Department of Physics Broida Hall, University of California, Santa Barbara, CA 93106, USA \\ ${ }^{19}$ NASA Exoplanet Science Institute, Caltech, MS 100-22, 770 South Wilson Avenue, Pasadena, CA 91125, USA \\ ${ }^{20}$ Center for Cosmology and Particle Physics, New York University, New York, NY, USA \\ ${ }^{21}$ Consejo Superior de Investigaciones Científicas, Calle Serrano, 117, E-28006 Madrid, Spain \\ 22 Instituto de Física, UFRGS, Caixa Postal 15051, Porto Alegre, RS 91501-970, Brazil \\ ${ }^{23}$ Indian Institute of Astrophysics, II Block, Koramangala, Bangalore 560 034, India \\ Received 2013 January 17; accepted 2013 July 11; published 2013 August 12
}

\begin{abstract}
We report the discovery of a candidate brown dwarf (BD) or a very low mass stellar companion (MARVELS-5b) to the star HIP 67526 from the Multi-object Apache point observatory Radial Velocity Exoplanet Large-area Survey (MARVELS). The radial velocity curve for this object contains 31 epochs spread over 2.5 yr. Our Keplerian fit, using a Markov Chain Monte Carlo approach, reveals that the companion has an orbital period of $90.2695_{-0.0187}^{+0.0188}$ days, an eccentricity of $0.4375 \pm 0.0040$, and a semi-amplitude of $2948.14_{-16.55}^{+16.65} \mathrm{~m} \mathrm{~s}^{-1}$. Using additional high-resolution spectroscopy, we find the host star has an effective temperature $T_{\text {eff }}=6004 \pm 34 \mathrm{~K}$, a surface gravity $\log g(\operatorname{cgs})$ $=4.55 \pm 0.17$, and a metallicity $[\mathrm{Fe} / \mathrm{H}]=+0.04 \pm 0.06$. The stellar mass and radius determined through the empirical relationship of Torres et al. yields $1.10 \pm 0.09 M_{\odot}$ and $0.92 \pm 0.19 R_{\odot}$. The minimum mass of MARVELS$5 \mathrm{~b}$ is $65.0 \pm 2.9 \mathrm{M}_{\mathrm{Jup}}$, indicating that it is likely to be either a $\mathrm{BD}$ or a very low mass star, thus occupying a relatively sparsely populated region of the mass function of companions to solar-type stars. The distance to this system is 101 $\pm 10 \mathrm{pc}$ from the astrometric measurements of Hipparcos. No stellar tertiary is detected in the high-contrast images taken by either FastCam lucky imaging or Keck adaptive optics imaging, ruling out any star with mass greater than $0.2 M_{\odot}$ at a separation larger than $40 \mathrm{AU}$.
\end{abstract}

Key words: binaries: spectroscopic - brown dwarfs - stars: individual (HIP 67526) - stars: low-mass - techniques: radial velocities

Online-only material: color figures

\section{INTRODUCTION}

Brown dwarfs (BDs; Basri 2000) are star-like objects that are not massive enough to sustain stable hydrogen burning but

\footnotetext{
${ }^{24}$ LAMOST Fellow.
}

are sufficiently massive to fuse deuterium (Chabrier et al. 2000; Spiegel et al. 2011). As a result, their luminosity and temperature drop throughout their lifetimes (e.g., Burrows et al. 1997; Baraffe et al. 2003). To date, over 800 BDs have been directly and indirectly discovered through a variety of methods (e.g., Rebolo et al. 1995; Oppenheimer et al. 1995; Ruiz et al. 1997; 
Tinney et al. 1997; Kirkpatrick et al. 1999, 2000, 2011; Marcy \& Butler 2000; Mayor \& Udry 2000; Sahlmann et al. 2011). Most of the known BDs are free-floating objects detected in the imaging surveys. These surveys seem to imply a continuous distribution of masses through the hydrogen burning limit, with the abundance of $\mathrm{BD}$ rivaling that of stars.

The radial velocity (RV) technique has rapidly developed in the last three decades, and has led to the first discoveries of extrasolar planets around solar-like stars (Latham et al. 1989; Mayor \& Queloz 1995; Marcy \& Butler 1996). Since the reflex RV semi-amplitudes induced by BD companions could be many hundreds of meters per second, which are considerably larger than the signals induced by planetary companions, RV surveys should easily discover BD companions. However, only $60 \mathrm{BD}$ companions to solar-like stars in relatively short ( $P<10^{4}$ days) orbits have been identified in all the previous RV surveys (e.g., Marcy \& Butler 2000; Mayor \& Udry 2000; Vogt et al. 2002; Sahlmann et al. 2011; Díaz et al. 2012). The distribution of masses for spectroscopic companions to solar-like stars shows a clear deficit in the BD mass range (the "brown dwarf desert"; Marcy \& Butler 2000), quite in contrast to the surveys of free-floating BDs. Moreover, the statistical investigations of stellar companions to solar-like stars have shown a paucity of companions with mass ratios $\left(q \equiv M_{c} / M_{\star}\right)<0.2$, suggesting that the short-period BD desert extends in mass toward the low-mass star regime (Pont et al. 2005; Burgasser et al. 2007; Bouchy et al. 2011; Wisniewski et al. 2012).

The Multi-object Apache Point Observatory (APO) Radial Velocity Exoplanet Large-area Survey (MARVELS), part of the Sloan Digital Sky Survey III (SDSS-III; Eisenstein et al. 2011) program, ${ }^{25}$ monitors several thousands of stars in the magnitude range $V=8-12$ by visiting each star $\sim 24$ times over an 18 month interval with moderate RV precision (Ge et al. 2008; Ge et al. 2009; Ge \& Eisenstein 2009). Currently, more than 10 very low mass stellar and substellar companion candidates have been identified. In order to confirm the discoveries and characterize them further, the MARVELS survey team made extensive follow-up observations, including high-precision RV monitoring, high-resolution spectroscopy, time-series photometry, and high-contrast imaging.

High-precision RV follow-up observations are useful to refine orbital solutions and to detect additional lower-mass companions in the candidate systems. We also used multi-epoch high-resolution spectroscopy to rule out potential false alarms due to spectral contamination at the moderate resolving power of the MARVELS spectrograph. MARVELS-1b was announced as the first detection of a BD candidate from MARVELS (Lee et al. 2011). Further analysis of precise RVs made with the Hobby-Eberly Telescope High Resolution Spectrograph initially suggested an interior giant planet in a 3:1 period commensurability with MARVELS-1b. However, the apparent $\mathrm{RV}$ residuals to a one-companion fit were later proven to be due to spectral contamination by a stellar companion. This was determined by the identification of strong line bisector variations (Wright et al. 2013). MARVELS-1 is actually a face-on doublelined spectroscopic binary instead of a single star with a BD companion. In another MARVELS candidate BD system (TYC 3010-1494-1), a highly eccentric, double-lined spectroscopic binary star system masqueraded as the RV signal of a single star orbited by a very low mass companion (Mack et al. 2013).

\footnotetext{
25 http://www.sdss3.org/surveys/marvels.php
}

Excluding these two false positive detections, three out of four published MARVELS discoveries have a possible tertiary companion detected at wide separations in their systems. MARVELS-2b is likely to be a low-mass stellar companion with a short-period orbit around the F star TYC 2930-00872-1 and a stellar tertiary is identified by analyzing the long-term trend in the RV curve (Fleming et al. 2012). For MARVELS-3b, a faint candidate tertiary companion is detected in the Keck adaptive optics (AO) image, separated by $\sim 1^{\prime \prime}$ from its host star (TYC 4110-01037-1; Wisniewski et al. 2012). Ma et al. (2013) detected a faint point source at a separation of $\sim 0$ '. 6 from the host star of MARVELS-4b (TYC 2087-00255-1) through high-contrast imaging. Future proper motion observations are necessary to resolve whether the offset objects are physically associated with the host stars. Nevertheless, these results have encouraged the MARVELS team to keep assessing the multiplicity for every future discovery in the survey.

Currently, there are about 60 BD companions to solar-like stars that have been reported in the literature. The distribution of masses of the companions exhibits a local minimum (the most "arid" part of the desert) in the mass range of $\sim 30-50 M_{\text {Jup }}$ (Sahlmann et al. 2011; Ma \& Ge 2013). The tentative bimodal distribution of mass may indicate that there are two formation mechanisms for $\mathrm{BD}$ and low-mass stellar companions: the low-mass BDs form by core accretion in protoplanetary disks while more massive companions form by gravitational collapse (Grether \& Lineweaver 2006; Sahlmann et al. 2011; Ma \& Ge 2013). Moreover, the properties of host stars might also have an important impact on the formation of BD companions. Bouchy et al. (2011) reported that super-Jupiters, BDs, and low-mass M dwarf companions $\left(10-100 M_{\text {Jup }}\right)$ to G-type $\left(T_{\text {eff }} \lesssim 6200 \mathrm{~K}\right)$ stars were apparently less common than similar companions to hotter stars. Compared to the metallicity of the planet hosts (Santos et al. 2001; Valenti \& Fischer 2005; Johnson et al. 2010), in general the hosts of BD companions are not that metal rich (Ma \& Ge 2013). Apparently the statistics of physical parameters are important for us to understand the formation and evolution of low-mass companions. Therefore, the MARVELS team has taken pains to conduct follow-up studies of MARVELS candidates in order to collect a uniformly characterized sample for a meta-analysis.

In this paper, we report a candidate BD or a low-mass stellar companion (MARVELS-5b) to HIP 67526 with a period of $\sim 90$ days from MARVELS. In Section 2.1, we describe the RV measurements and solve for the spectroscopic orbital elements using Markov Chain Monte Carlo (MCMC) analysis. We analyze the photometric data from SuperWASP and the astrometric data from Hipparcos in Sections 2.2 and 2.3, respectively. In Section 3.1, we determine precise stellar parameters for the primary star. Using the stellar mass derived in Section 3.2, we then estimate the mass of the companion in Section 3.3. The evolutionary state of the host star is studied in Section 3.4. The high-contrast imaging is presented in Section 3.5. Finally, we provide a discussion and a summary in Section 4.

\section{OBSERVATIONS AND RESULTS FOR THE LOW-MASS COMPANION}

\subsection{Differential Radial Velocities}

\subsubsection{MARVELS and TNG/SARG Measurements}

HIP 67526 was selected as an RV survey target according to the MARVELS preselection criterion (Lee et al. 2011). It has been monitored at 21 epochs using the MARVELS 


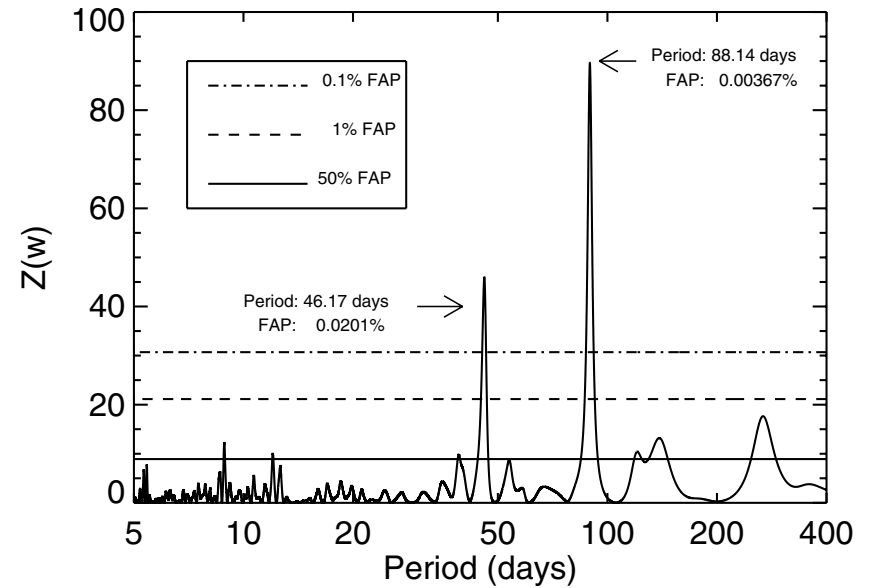

Figure 1. Periodogram for MARVELS RV measurements of HIP 67526 exhibiting two peaks at periods of $\sim 88$ days and $\sim 46$ days. The three horizontal lines indicate the false alarm probability at different levels $(50 \%, 1 \%, 0.1 \%)$.

instrument mounted on the SDSS $2.5 \mathrm{~m}$ Telescope at APO (Gunn et al. 2006) during the first two-year cycle of the SDSS-III MARVELS planet-search program (Ge et al. 2008). The MARVELS instrument is a fiber-fed dispersed fixeddelay interferometer instrument capable of observing 60 objects simultaneously, designed for a large-scale RV survey (Ge 2002; Ge et al. 2009). The dispersed fixed-delay interferometer instrument principle is described in several prior papers $(\mathrm{Ge}$ 2002; Ge et al. 2002; Erskine 2002; Erskine et al. 2003; Ge et al. 2006; van Eyken et al. 2011; Wang et al. 2011). The MARVELS interferometer delay calibration is described by Wang et al. (2012a, 2012b). The interferometer produces two fringing spectra per object, covering a wavelength range of 5000-5700 , with a resolving power of $R \sim 12,000$. Two iodine absorption spectra of light from a tungsten lamp taken before and after each science exposure are used to calibrate any instrument drift. Data processing and the error estimation algorithm have been described in detail by Lee et al. (2011) and Fleming et al. (2010), respectively.
HIP 67526 was identified as a star bearing an unseen companion by performing Lomb-Scargle (L-S) periodogram analysis (e.g., Lomb 1976; Scargle 1982; Cumming 2004; Baluev 2008) on the 21 MARVELS RV points. There are two significant peaks on the L-S periodogram with periods at $\sim 88$ days and $\sim 46$ days (Figure 1 ). The false alarm probability (hereafter FAP) of the 88 day peak is $0.00367 \%$, and the FAP of the 46 day peak is $0.0201 \%$. We fit a Keplerian orbit to the observed RV curve, forcing the period to be close to $\sim 88$ days and $\sim 46$ days. The preliminary fitting results are illustrated in Figure 2. The solution at an orbital period of 90.2 days provides a better fit to the MARVELS RV curve than the solution at an orbital period of 45.6 days. The shorter orbital period peak in the periodogram is probably an alias. The minimum mass (if $\sin i=1$ ) of the unseen companion from the longer period solution is $\sim 65 M_{\text {Jup }}$ (see Section 3.3 for details). The estimated minimum mass is below the hydrogen burning limit and places MARVELS-5b within the sparsely populated region of the mass function of companions to solar-like stars.

We collected 10 additional RV measurements with the SARG spectrograph (Gratton et al. 2001) at the $3.58 \mathrm{~m}$ Telescopio Nazionale Galileo (TNG) Telescope in late 2010 and 2011. The spectrograph covers a wavelength range of 4620-7920 A with $R \sim 57,000$. The simultaneous iodine cell technique (Butler et al. 1996) was employed to calibrate the RV measurements. The raw spectra were reduced by using the standard IRAF ${ }^{26}$ Echelle reduction packages. The final extracted differential RVs from MARVELS and TNG/SARG are presented in Table 1. The RV curve was sampled in total at 31 epochs using these two instruments over $2.5 \mathrm{yr}$.

\subsubsection{Spectroscopic Orbital Elements}

We have performed a Bayesian analysis of the observed RVs using a model consisting of the primary star and one low-mass companion on an eccentric Keplerian orbit based on

26 http://iraf.noao.edu/

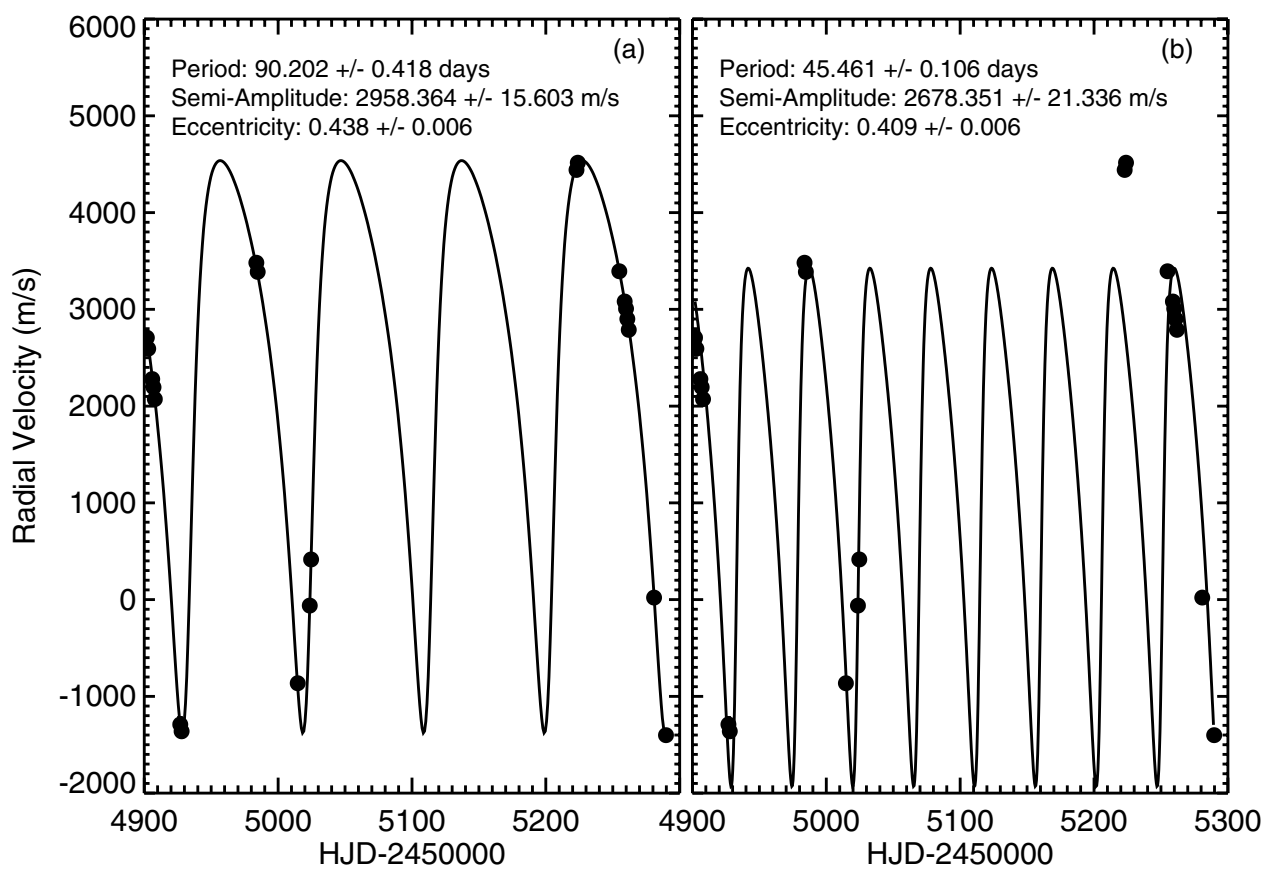

Figure 2. Keplerian fitting results for the MARVELS RV measurements of HIP 67526 forcing the period close to $\sim 88$ days (panel a) and $\sim 46$ days (panel b). 
Table 1

Differential Radial Velocity Measurements

\begin{tabular}{|c|c|c|c|}
\hline Instrument & $\mathrm{HJD}^{\mathrm{a}}$ & $\begin{array}{c}\mathrm{RV} \\
\left(\mathrm{m} \mathrm{s}^{-1}\right)\end{array}$ & $\begin{array}{c}\text { Error } \\
\left(\mathrm{m} \mathrm{s}^{-1}\right)\end{array}$ \\
\hline \multirow[t]{21}{*}{ MARVELS } & 2454901.933194 & 2707.27 & 59.93 \\
\hline & 2454902.932454 & 2593.08 & 36.46 \\
\hline & 2454905.940208 & 2279.35 & 37.21 \\
\hline & 2454906.909213 & 2194.67 & 33.47 \\
\hline & 2454907.900972 & 2072.03 & 26.32 \\
\hline & 2454926.884479 & -1290.14 & 71.20 \\
\hline & 2454927.893461 & -1360.84 & 58.21 \\
\hline & 2454983.735220 & 3481.73 & 29.43 \\
\hline & 2454984.726944 & 3387.25 & 33.10 \\
\hline & 2455014.633588 & -864.37 & 33.17 \\
\hline & 2455023.635463 & -61.99 & 30.11 \\
\hline & 2455024.635984 & 414.89 & 38.37 \\
\hline & 2455222.898646 & 4441.05 & 36.96 \\
\hline & 2455223.889306 & 4515.50 & 33.08 \\
\hline & 2455254.894387 & 3393.48 & 33.77 \\
\hline & 2455258.906053 & 3081.05 & 31.48 \\
\hline & 2455259.892963 & 3006.84 & 29.68 \\
\hline & 2455260.882778 & 2900.49 & 33.60 \\
\hline & 2455261.939502 & 2788.23 & 31.63 \\
\hline & 2455280.845833 & 20.73 & 37.47 \\
\hline & 2455289.750139 & -1401.23 & 33.69 \\
\hline \multirow[t]{10}{*}{ TNG/SARG } & 2455553.785137 & -3334.74 & 8.97 \\
\hline & 2455580.668228 & 1340.35 & 8.38 \\
\hline & 2455580.692489 & 1359.08 & 10.57 \\
\hline & 2455666.497671 & 873.73 & 9.94 \\
\hline & 2455666.521154 & 878.94 & 10.24 \\
\hline & 2455698.415610 & 998.61 & 9.27 \\
\hline & 2455725.369700 & -1587.03 & 10.94 \\
\hline & 2455760.380520 & 1250.09 & 10.09 \\
\hline & 2455791.354961 & 779.89 & 12.41 \\
\hline & 2455791.378282 & 760.40 & 13.30 \\
\hline
\end{tabular}

Note. ${ }^{a}$ Heliocentric Julian Day.

the combined differential RV observations of MARVELS and TNG/SARG.

We calculated a posterior sample using the MCMC technique as described in Ford (2006). Each state in the Markov Chain is described by the parameter set $\boldsymbol{\theta}=$ $\left\{P, K, e, \omega, M, \gamma_{M}, \gamma_{T}, \sigma_{j}\right\}$, where $P$ is the orbital period, $K$ is the velocity semi-amplitude, $e$ is the orbital eccentricity, $\omega$ is the argument of periastron, and $M$ is the mean anomaly at the chosen epoch $(\tau)$. The parameters $\gamma_{M}$ and $\gamma_{T}$ are constant systemic velocity terms for the MARVELS and TNG/SARG instruments, respectively, used to account for the offsets between the observed differential RV data and the zero point of the Keplerian RV model. The "jitter" parameter, $\sigma_{j}$, describes any excess noise (Wright 2005), including both astrophysical sources of noise (e.g., stellar oscillation, stellar spots) and any instrumental noise not accounted for in the quoted measurement uncertainties. We use standard priors for each parameter (see Ford \& Gregory 2007). The prior is uniform in the log of the orbital period $P$, while for $K$ and $\sigma_{j}$ we used a modified Jeffery's prior (Gregory 2005). Priors for the remaining parameters are uniform: $e$ (between zero and unity), $\omega$ and $M$ (between zero and $2 \pi), \gamma_{M}$ and $\gamma_{T}$. Following Ford (2006), we adopt a likelihood (i.e., conditional probability of making the specified measurements given a particular set of model parameters) of

$$
p(v \mid \boldsymbol{\theta}, M) \propto \prod_{k} \frac{\exp \left[-\left(v_{k, \theta}-v_{k}\right)^{2} / 2 \sigma_{k}^{2}\right]}{\sqrt{\sigma_{j}^{2}+\sigma_{k}^{2}}},
$$

Table 2

Orbital Elements of MARVELS-5b

\begin{tabular}{llc}
\hline \hline Parameter & \multicolumn{1}{c}{ Units } & Value \\
\hline$P$ & Period (days) & $90.2695_{-0.0187}^{+0.0188}$ \\
$K$ & RV semi-amplitude $\left(\mathrm{m} \mathrm{s}^{-1}\right)$ & $2948.14_{-16.55}^{+16.65}$ \\
$e$ & Eccentricity & $0.4375 \pm 0.0040$ \\
$\omega$ & Argument of periastron (deg) & $-140.91 \pm 0.54$ \\
$T_{0}$ & Epoch of periastron (HJD) & $2455563.73 \pm 0.20$ \\
$\gamma_{M}$ & MARVELS systemic velocity $\left(\mathrm{m} \mathrm{s}^{-1}\right)$ & $2573.71 \pm 11.31$ \\
$\gamma_{T}$ & TNG/SARG systemic velocity $\left(\mathrm{m} \mathrm{s}^{-1}\right)$ & $-374.69 \pm 11.4$ \\
$\sigma_{j}$ & Jitter $\left(\mathrm{m} \mathrm{s}^{-1}\right)$ & $21.35_{-4.97}^{+6.47}$ \\
\hline
\end{tabular}

where $v_{k}$ is observed velocity at time $t_{k}, v_{k, \theta}$ is the model velocity at time $t_{k}$ given the model parameters $\boldsymbol{\theta}$, and $\sigma_{k}$ is the measurement uncertainty for the observation at time $t_{k}$.

To test the robustness of the MCMC analysis, we calculate five Markov Chains starting from different initial states, each for $5 \times 10^{7}$ states. To prevent the choice of initial states from influencing our results, we consider only the second half of each chain. We calculate the Gelman-Rubin test statistic (which compares the variance of a parameter within each chain to the variance between chains; Gelman \& Rubin 1992) for each model parameter. We find no indications that the Markov Chains have yet to converge and conclude that the Markov Chains provide an adequate posterior sample for inferring the orbital parameters and uncertainties.

We combine the Markov Chains described above to estimate the joint posterior probability distribution for the orbital model of HIP 67526. For orbital eccentricity, we also used the $\Gamma$ method described in Wang \& Ford (2011), which leads to a result similar to that from the MCMC analysis. The median values are taken for each model parameter based on the marginal posterior probability distributions. The uncertainties are calculated as the standard deviation about the mean value from the combined posterior sample. Since the shape of the marginal posterior distribution is roughly similar to a multivariate normal distribution, the median value plus or minus the reported uncertainty roughly corresponds to a $68.3 \%$ confidence interval. Finally, we convert the model parameters to traditional standard parameters of a spectroscopic orbit and report the results in Table 2. The phase-folded RV curve is presented in Figure 3.

\subsection{SuperWASP Photometry}

We searched the SuperWASP public archived database (Butters et al. 2010) and found 1378 photometric data measurements of HIP 67526 observed in 2004 and 5680 data points in 2007. The mean absolute deviation of the light curve is $9.7 \mathrm{mmag}$. We first searched for a transit-like dip in brightness at short periods between 0.2 and 10 days. We find no significant detection of a transit event. Next, we searched for transits specifically in the range of 85-95 days, which includes the bestfit period from the spectroscopic RV curve. The phase-folded data are sparsely covered at these long periods, and we find no significant transit signal. In summary, we do not find a transit in SuperWASP photometric data with a long or a short period. We also attempted to search for a sinusoidal signal in the light curve but found no significant signal.

\subsection{Hipparcos Astrometry}

HIP 67526 exists in the Hipparcos catalog with a parallax distance of $100 \pm 10 \mathrm{pc}$ from the Sun. It is possible that the orbital motion of the star due to the gravitational influence of 


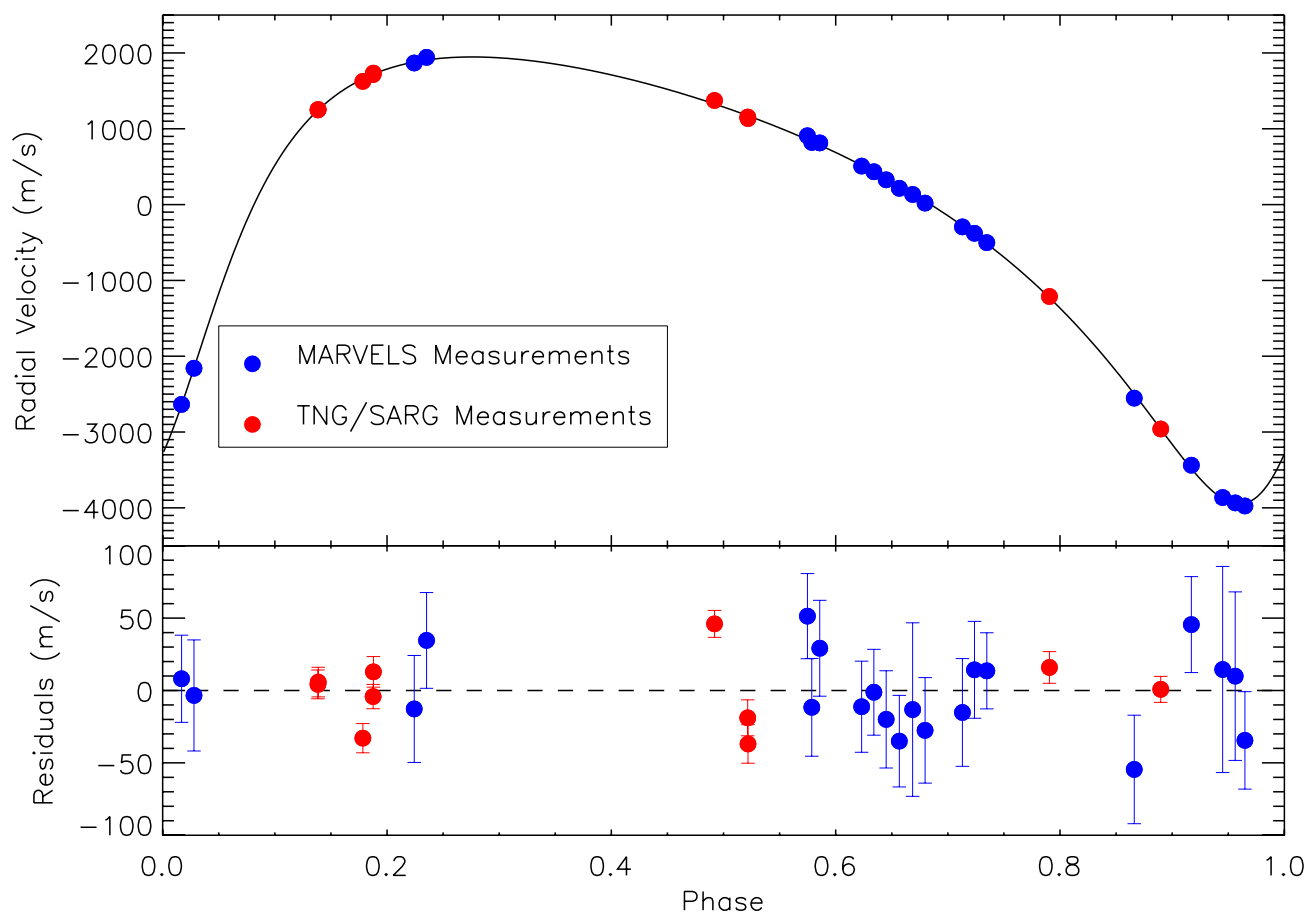

Figure 3. Phase-folded RV curve for MARVELS-5b, having a period of $90.2695_{-0.0187}^{+0.0188}$ days, an eccentricity of $0.4375 \pm 0.0040$, and a semi-amplitude of

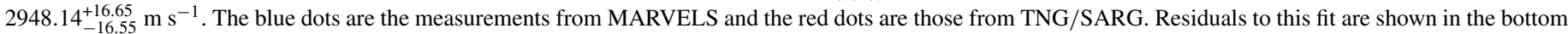
panel.

(A color version of this figure is available in the online journal.)

its companion can be resolved by Hipparcos astrometry. This would allow the inclination $i$ and the ascending node $\Omega$ of the Keplerian orbit, and thus the true mass of MARVELS-5b, to be well constrained (Sahlmann et al. 2011). We retrieved the dataset of HIP 67526 from the Intermediate Astrometric Data (IAD) of the new Hipparcos reduction (van Leeuwen 2007), including the satellite orbit number, the epoch $t$, the parallax factor $\Pi$, the scan angle orientation $\psi$, the abscissa residual $\delta \Lambda$, and the abscissa error $\sigma_{\Lambda}$ for every satellite scan. There are 123 available Hipparcos scans on HIP 67526 in the IAD and the average abscissa error is $\overline{\sigma_{\Lambda}} \sim 10$ mas. Thus, the dataset allows a $1 \sigma$ detection of an orbit with an angular size of $\overline{\sigma_{\Lambda}} / \sqrt{N}=10 / \sqrt{123} \sim 1$ mas.

We then estimate the minimum angular semimajor axis (in mas) of the primary's orbit, which can be written as

$$
a_{a} \sin i=3.35729138 \times 10^{-5} K P \sqrt{1-e^{2}} \varpi,
$$

where $K$ (in $\mathrm{m} \mathrm{s}^{-1}$ ), $P$ (in yr), and $e$ are the spectroscopic orbital elements, $\varpi$ (in mas) is the parallax, and $i$ is the unknown inclination (Pourbaix 2001). This equation yields a minimum angular semimajor axis $\sim 0.2$ mas for HIP 67526 . Therefore, for nearly edge-on orbits, the angular size of the primary's orbit is well below the $1 \sigma$ detection threshold, and thus the motion of HIP 67526 about the system's center-of-mass cannot be detected for such geometries. Assuming that the Hipparcos data of HIP 67526 are consistent with no astrometric signal from the orbit around the center-of-mass of the system, and that orbits of $\sim 1$, $\sim 2$, and $\sim 3$ mas would have been detected at $1 \sigma, 2 \sigma$, and $3 \sigma$, we can place upper limits on the companion mass of $\sim 0.33 M_{\odot}$ $(1 \sigma)$, and $\sim 0.80 M_{\odot}(2 \sigma)$, and $1.49 M_{\odot}(3 \sigma)$. As argued in Section 3.3, such massive companions are a posteriori unlikely to be flat or falling priors on the companion mass distribution. For priors that increase with increasing mass, companions of mass $\gtrsim 0.5 M_{\odot}$ are not a posteriori implausible, but would be ruled out based on the lack of evidence of a second set of spectral lines in the high-resolution spectra, if the companion was luminous (i.e., not a remnant).

\section{OBSERVATIONS AND RESULTS FOR THE HOST STAR}

\subsection{Spectroscopic Parameters and Spectral Energy Distribution Analysis}

In order to characterize the host star HIP 67526, two moderate-resolution spectra $(R \sim 31,500)$ were taken with the ARC Echelle Spectrograph (ARCES; Wang et al. 2003) mounted on the Apache Point Observatory $3.5 \mathrm{~m}$ telescope on UT 2010 June 10. The spectra cover the full optical range from $3600 \AA$ to $1.0 \mu \mathrm{m}$. The spectra were obtained using the default 1 .. $6 \times 3$ 3.' 2 slit and an exposure time of $1200 \mathrm{~s}$. The raw data were processed using standard IRAF techniques. The extracted one-dimensional spectra were converted to vacuum wavelengths and to the heliocentric frame. The data were normalized by fitting a series of polynomials to the continuum.

We utilized two individual pipelines to derive basic stellar parameters such as $T_{\text {eff }}, \log g$, and $[\mathrm{Fe} / \mathrm{H}]$ for the host star. Both pipelines are based on the requirements of excitation and ionization equilibria of Fe I and Fe II. However, different versions of ATLAS9 plane-parallel model atmospheres (Kurucz 1993 and Castelli \& Kurucz 2004) and different iteration algorithms are implemented. We refer the readers to Wisniewski et al. (2012) for more details on the pipelines. The derived stellar parameters from these two pipelines are usually consistent to within $1 \sigma$ of the associated errors. Thus, we simply adopted the weighted average values as the final determined stellar parameters. We combined the internal errors from the two pipelines as $1 / \sigma^{2}=1 / \sigma_{1}^{2}+1 / \sigma_{2}^{2}$ for each parameter, and 
Table 3

Stellar Parameters of HIP 67526

\begin{tabular}{|c|c|c|}
\hline Parameter & Result & Note \\
\hline$B$ & $10.303 \pm 0.032 \mathrm{mag}$ & Kharchenko \& Roeser (2009) \\
\hline$V$ & $9.706 \pm 0.027 \mathrm{mag}$ & Kharchenko \& Roeser (2009) \\
\hline$J$ & $8.598 \pm 0.020 \mathrm{mag}$ & 2MASS \\
\hline$H$ & $8.363 \pm 0.049 \mathrm{mag}$ & 2MASS \\
\hline$K$ & $8.295 \pm 0.024 \mathrm{mag}$ & 2MASS \\
\hline$W 1$ & $8.226 \pm 0.022 \mathrm{mag}$ & WISE \\
\hline$W 2$ & $8.283 \pm 0.020 \mathrm{mag}$ & WISE \\
\hline$W 3$ & $8.260 \pm 0.017 \mathrm{mag}$ & WISE \\
\hline$W 4$ & $8.063 \pm 0.150 \mathrm{mag}$ & WISE \\
\hline$T_{\text {eff }}$ & $6004 \pm 34 \mathrm{~K}$ & Spectroscopy \\
\hline $\log g(\mathrm{cgs})$ & $4.55 \pm 0.17$ & Spectroscopy \\
\hline$[\mathrm{Fe} / \mathrm{H}]$ & $+0.04 \pm 0.06$ & Spectroscopy \\
\hline$V_{\text {mic }}$ & $1.03 \pm 0.04 \mathrm{~km} \mathrm{~s}^{-1}$ & Spectroscopy \\
\hline$M_{\star}$ & $1.10 \pm 0.09 M_{\odot}$ & Torres et al. (2010) \\
\hline$R_{\star}$ & $0.92 \pm 0.19 R_{\odot}$ & Torres et al. (2010) \\
\hline$A_{\mathrm{V}}$ & $0.035 \pm 0.035 \mathrm{mag}$ & SED Fitting \\
\hline$\varpi$ & $9.87 \pm 1.26 \mathrm{mas}$ & Hipparcos \\
\hline$M_{\star}$ & $1.11 \pm 0.08 M_{\odot}$ & MCMC \\
\hline$R_{\star}$ & $0.95_{-0.14}^{+0.15} R_{\odot}$ & $\mathrm{MCMC}$ \\
\hline$T_{\text {eff }}$ & $6004 \pm 34 \mathrm{~K}$ & MCMC \\
\hline $\log g(\mathrm{cgs})$ & $4.53_{-0.13}^{+0.15}$ & $\mathrm{MCMC}$ \\
\hline$[\mathrm{Fe} / \mathrm{H}]$ & $+0.04 \pm 0.06$ & MCMC \\
\hline$\varpi$ & $10.25_{-1.10}^{+1.09} \mathrm{mas}$ & MCMC \\
\hline$A_{\mathrm{V}}$ & $0.043_{-0.027}^{+0.033} \mathrm{mag}$ & $\mathrm{MCMC}$ \\
\hline
\end{tabular}

added in quadrature a systematic error of $18 \mathrm{~K}, 0.08,0.03$, and $0.02 \mathrm{~km} \mathrm{~s}^{-1}$ for $T_{\text {eff }}, \log g,[\mathrm{Fe} / \mathrm{H}]$, and $V_{\text {mic }}$, respectively (Wisniewski et al. 2012). The final results are summarized in Table 3.

We collected the optical and near-infrared absolute photometry of HIP 67526 from the Hipparcos, Two Micron All Sky Survey (2MASS), and Wide-field Infrared Survey Explorer (WISE) catalogs (Table 3) to construct a spectral energy distribution (SED; see Figure 4) and fit it with a NextGen model atmosphere (Hauschildt et al. 1999). The resultant stellar parameters, $T_{\text {eff }}=5800 \pm 200 \mathrm{~K}, \log g(\mathrm{cgs})=4.0 \pm 1.0$ and $[\mathrm{Fe} / \mathrm{H}]=0.0 \pm 0.5$, are in good agreement with the parameters derived from spectroscopy within the errorbars. In addition, the SED fitting indicates that HIP 67526 suffers only slight extinction $\left(A_{\mathrm{V}}=0.035 \pm 0.035\right)$.

\subsection{Stellar Mass and Radius}

We determine the stellar mass and radius using two methods. First, we use the empirical relationship of Torres et al. (2010) with our values for $T_{\text {eff }}, \log g$, and $[\mathrm{Fe} / \mathrm{H}]$. Uncertainties in the mass and radius are derived by adding in quadrature the correlations of the best-fit coefficients from Torres et al. (2010) and the scatter in the relation as reported in their study. The correlations between the stellar parameters $T_{\text {eff }}, \log g$, and $[\mathrm{Fe} / \mathrm{H}]$ are not measured and are therefore not considered. We find a mass $M_{\star}=1.10 \pm 0.09 M_{\odot}$ and a radius $R_{\star}=$ $0.92 \pm 0.19 R_{\odot}$.

The existence of a trigonometric parallax provides additional information to constrain the mass and radius of the primary star. We incorporate this data by running an MCMC analysis that fully explores parameter space. One million iterations in the MCMC were run, stepping through $T_{\text {eff }}, \log g$, $[\mathrm{Fe} / \mathrm{H}]$, parallax $(\varpi)$, and $A_{\mathrm{V}}$. We use random starting values to initiate the chain. For each iteration, we calculate a mass and

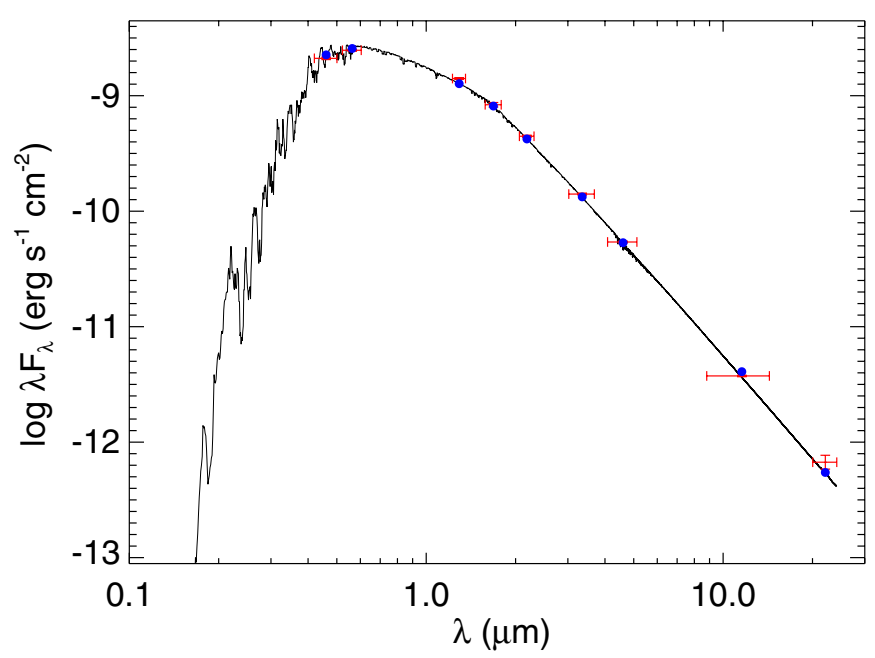

Figure 4. Observed SED for HIP 67526 overplotted with the best-fit NextGen model atmosphere emission. Blue points represent the expected fluxes in each band based on the best-fit model, red horizontal bars are the bandpass widths, and red vertical bars are the uncertainties of measured fluxes. The resultant stellar parameters from this fit agreed to within $1 \sigma$ with the stellar parameters determined from analysis of moderate-resolution ARCES spectra.

(A color version of this figure is available in the online journal.)

radius following Torres et al. (2010) and the iteration's values of $T_{\text {eff }}, \log g$, and [Fe/H]. A stellar luminosity is calculated via the Stefan-Boltzmann law, then a bolometric correction to the 2MASS $\mathrm{K}_{\mathrm{s}}$ band is applied by interpolating the table of corrections as a function of $T_{\text {eff }}$ for $[\mathrm{M} / \mathrm{H}]=0.0$ and $\log g=4.5$ from Masana et al. (2006). The absolute $K_{\mathrm{s}}$ magnitude is calculated from the luminosity and bolometric correction, after which the apparent magnitude is calculated from the absolute magnitude and the iteration's values of $\varpi$ and $A_{\mathrm{V}}$.

After each iteration, a $\chi^{2}$ statistic is calculated as the sum of the individual $\chi^{2}$ for $T_{\text {eff }}, \log g,[\mathrm{Fe} / \mathrm{H}], \varpi$, and $A_{\mathrm{V}}$, where the expected values for $T_{\text {eff }}, \log g$, and $[\mathrm{Fe} / \mathrm{H}]$ are the values determined spectroscopically, the expected value for $\varpi$ comes from the Hipparcos catalog, and the expected value for $A_{\mathrm{V}}$ comes from the SED analysis. The next iteration's trial parameters are selected using Gaussians centered on the current iteration's values with widths equal to the $1 \sigma$ parameter uncertainties for $T_{\mathrm{eff}},[\mathrm{Fe} / \mathrm{H}]$, and $A_{\mathrm{V}}$, and $0.1 \sigma$ for $\log g$ and $\varpi$. These widths were empirically determined such that the overall trial acceptance rate was $\sim 24 \%$, close to the optimal value for multi-dimensional chains (Gelman et al. 2003).

The first $1 \%$ of iterations are rejected as a burn-in period, while the remaining iterations are used to determine the bestfit final parameters $\left(M_{\star}, R_{\star}, T_{\text {eff }}, \log g,[\mathrm{Fe} / \mathrm{H}], \varpi, A_{\mathrm{V}}\right)$. The $1 \sigma$ uncertainties are derived based on the cumulative histogram of each parameter. For the stellar mass and radius uncertainties, the reported scatter in Torres et al. (2010) is also added in quadrature. Each parameter agrees to within $1 \sigma$ of the spectroscopic/SED/catalog values, and these parameters are tabulated in Table 3.

\subsection{Mass of the Candidate Low-mass Companion}

Using the spectroscopic orbital elements from the RV fit, we can derive the mass function of the companion,

$$
M_{f} \equiv \frac{\left(M_{c} \sin i\right)^{3}}{\left(M_{\star}+M_{c}\right)^{2}}=\frac{K^{3}\left(1-e^{2}\right)^{3 / 2} P}{2 \pi G},
$$




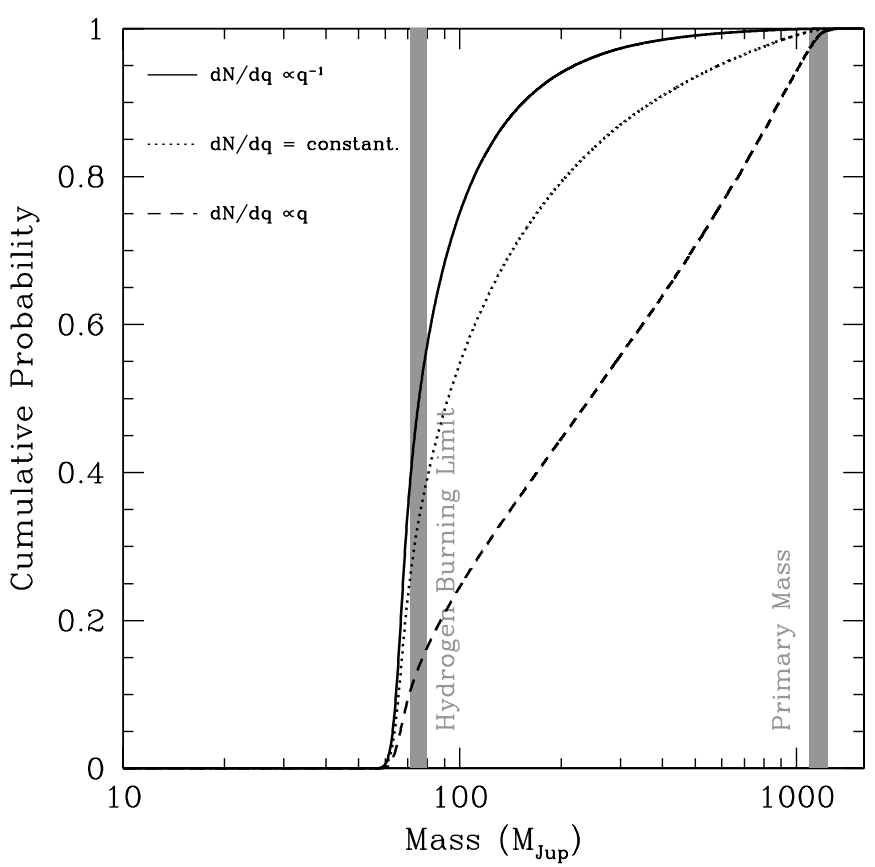

Figure 5. Cumulative probability that the mass of MARVELS-5b is less than a given mass for three priors on the companion mass ratio: $d N / d q \propto q^{-1}$ (solid line), $d N / d q=$ constant (dotted line), and $d N / d q \propto q^{+1}$ (dashed line).

which is independent of the mass of the primary and the inclination of orbit. For MARVELS-5b, we obtain

$$
M_{f}=(1.742 \pm 0.026) \times 10^{-4} M_{\odot},
$$

where the uncertainty is essentially dominated by the uncertainty in $K$ (see Table 2). Assuming $\sin i=1$, we derive its minimum mass $M_{\min }=65.0 \pm 2.9 M_{\text {Jup }}$. The uncertainty here is dominated by the uncertainty in the primary mass (see Table 3). We also find the minimum mass ratio of the companion $q_{\text {min }}=0.0560 \pm 0.0015$.

The true mass of the companion depends on the inclination of its orbit, which is unknown. We can estimate the posterior probability distribution of the true mass, assuming an isotropic distribution of orbits and adopting a prior for the distribution of the companion mass ratios. We therefore consider three reasonable priors on the companion mass ratio of the form: $d N / d q \propto q^{\alpha}$, where $\alpha=-1,0,+1$ (e.g., Grether \& Lineweaver 2006). The estimation was realized by using an MCMC, which has been described in detail in Fleming et al. (2010) and Lee et al. (2011). All sources of uncertainty from the mass function and the primary mass have been considered appropriately. We draw values of $\cos i$ from a uniform distribution and weight the resulting distribution by $q^{\alpha+1}$ in order to account for the mass ratio prior. For $\alpha>0$, the a posteriori distribution does not converge. However, we can rule out mass ratios $q>1$ for mainsequence companions by the lack of a second set of spectral lines in the high-resolution spectra. We therefore enforce $q \leqslant 1$, thus implicitly assuming the companion is not a stellar remnant. The resultant cumulative distributions of the true companion mass are presented in Figure 5, and we summarize the median mass as well as the transit probability for each of our priors in Table 4. For $\alpha<0$, MARVELS-5b is more likely to be a true $\mathrm{BD}$; for $\alpha=0$ or $\alpha=1$, it is more likely to be a low-mass stellar companion.

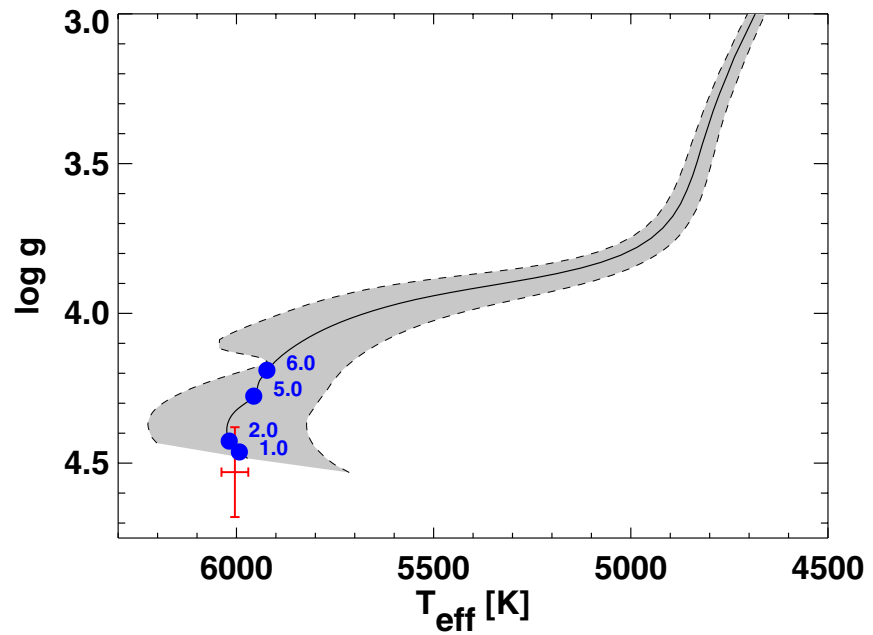

Figure 6. Comparison of the observed stellar parameters of HIP 67526 with a Yonsei-Yale stellar evolutionary track (Demarque et al. 2004) for an analogous star with $M_{\star}=1.10 M_{\odot}$ and $[\mathrm{Fe} / \mathrm{H}]=+0.04$. The shaded region indicates the $1 \sigma$ deviations in the evolutionary track. The blue dots are the location of the analogous star at different ages in Gyr. HIP 67526 (in red) is most likely a main-sequence dwarf star younger than $\sim 2.5 \mathrm{Gyr}$, judging by the evolutionary data alone, since most of the area within the $1 \sigma$ ellipsoid lies close to the ZAMS, but its low level of activity suggests an age over $\sim 3 \mathrm{Gyr}$, and thus it is most likely a middle-aged star.

(A color version of this figure is available in the online journal.)

Table 4

Companion Mass for Different Priors

\begin{tabular}{lcc}
\hline \hline Assumed Prior & Median Mass & Transit Prob. \\
\hline None $(\sin i=1)$ & $65.0 M_{\text {Jup }}$ & $100 \%$ \\
$d N / d q \propto q^{-1}$ & $75.6 M_{\text {Jup }}$ & $1.1 \%$ \\
$d N / d q=$ const & $95.3 M_{\text {Jup }}$ & $0.7 \%$ \\
$d N / d q \propto q^{+1}$ & $243.8 M_{\text {Jup }}$ & $0.2 \%$ \\
\hline
\end{tabular}

\subsection{Evolutionary State of the Host Star}

We estimate the evolutionary state of the host star HIP 67526 by comparing the measured stellar parameters with a Yonsei-Yale stellar evolutionary track (Demarque et al. 2004) for an analogous star with $M_{\star}=1.10 M_{\odot}$ and $[\mathrm{Fe} / \mathrm{H}]=$ 0.04. The result is displayed in Figure 6. The dashed curves represent the same evolutionary track but for stellar masses $\pm 0.08 M_{\odot}$, which is the $1 \sigma$ uncertainty in the stellar mass from the Torres et al. (2010) relation. The shaded region indicates the $1 \sigma$ deviations in the evolutionary track. The blue dots are the location of the star at different ages. The evolutionary data suggest a young star since most of the area of the $1 \sigma$ ellipsoid lies either below or very close to the zero-age main sequence (ZAMS). However, using the APO spectroscopic data, we measure the flux in the line cores of the $\mathrm{Ca}$ II $\mathrm{H}$ and $\mathrm{K}$ lines and calculate the activity index $\log \left(R_{\mathrm{HK}}^{\prime}\right)$, yielding -4.9 . This value points to an age of at least $\sim 3$ Gyr (e.g., Figure 11 of Mamajek \& Hillenbrand 2008). Both the H-R diagram and the HK activity levels, however, are poor age discriminants in this range of parameters. Taken together, the evolutionary and activity data point to a star no younger than 2-3 Gyr, and probably no older than the Sun, a range compatible with both criteria within the rather large errors. This range corresponds to our best estimate of the age of HIP 67526. 


\subsection{Direct Imaging Search for Visual Companions}

\subsubsection{FastCam Lucky Imaging}

Lucky imaging (LI, observations taken at very high cadence to achieve nearly diffraction-limited images from a subsample of the total) was performed using FastCam (Oscoz et al. 2008) on the 1.5 m Carlos Sánchez Telescope (TCS) at Observatorio del Teide in Spain. The primary goal of these observations was to search for companions at large separations that could contaminate spectroscopic observations of the target masquerading as a systematic trend in the RV data (Fleming et al. 2012). The LI frames were acquired on 2011 April 3, 2011 May 5, and 2011 May 8 in the $I$ band and spanning $\sim 21^{\prime \prime} \times 21^{\prime \prime}$ on the sky. On 2011 April 3 a total of 100,000 short-exposure images, each corresponding to $35 \mathrm{~ms}$ exposure time were acquired, on 2011 May 5 a total of 45,000 short-exposure images, each corresponding to $35 \mathrm{~ms}$ exposure time were acquired, and on 2011 May 8 a total of 45,000 short-exposure images, each corresponding to $50 \mathrm{~ms}$ exposure time were acquired. The data were processed using a custom IDL software pipeline. After identifying frames corrupted due to cosmic rays, electronic glitches, etc., the remaining frames were bias corrected and flat fielded.

LI selection was applied using a variety of selection thresholds (best $X \%$ ) based on the brightest pixel (BP) method. The selected BP must be below a specified brightness threshold to avoid selecting cosmic rays or other non-speckle features. As a further check, the BP must be consistent with the expected energy distribution from a diffraction speckle under the assumption of a diffraction-limited point-spread function (PSF). The BPs of each frame are then sorted from brightest to faintest, and the best $X \%$ are then shifted and added to generate a final image. In Figure 7 , we show the results of the LI selection and shift-and-add for different LI thresholds ranging from considering only the best $1 \%$ of the frames up to including $80 \%$ of the data for data collected in 2011 April and 2011 May. Each panel covers $\sim 5^{\prime \prime} .5 \times 5^{\prime \prime} .5$ centered on HIP 67526. Restricting the LI selection to the top percentage (i.e., the $1 \%$ LI image) improves the angular resolution with respect to choosing a lower threshold (i.e., the $80 \% \mathrm{LI}$ image) but at the cost of higher noise at large distances from the target.

We follow the same procedure as in Femenía et al. (2011) to compute the $3 \sigma$ detectability $(\Delta m)$ curves on each of the images whose $\sim 5^{\prime \prime} .5 \times 5^{\prime \prime} .5$ region around HIP 67526 has been depicted in Figure 7: at a given angular distance $\rho$ from HIP 67526 we identify all possible sets of small boxes of a size larger but comparable to the FWHM of the PSF (i.e., $5 \times 5$ pixel boxes). Only regions of the image showing structures easily recognizable as spikes due to diffraction of the telescope spider and/or artifacts on the read-out of the detector are dismissed. For each of the valid boxes on the arc at angular distance $\rho$ the standard deviation of the image pixels within the $5 \times 5$ pixel boxes is computed. The value assigned to the $3 \sigma$ detectability curve at $\rho$ is three times the mean value from the standard deviations of all the eligible boxes at $\rho$. This procedure on each of the LI \% thresholding values (in steps of $1 \%$ ) produces a detectability curve, while the envelope of the entire family of curves for a given night yields the best possible detectability curve to be extracted from the whole data set. These "best LI curves" for each of the three nights are depicted in Figure 8, where we can see the data collected are similar in quality to the data on May 8 providing slightly better contrast values.

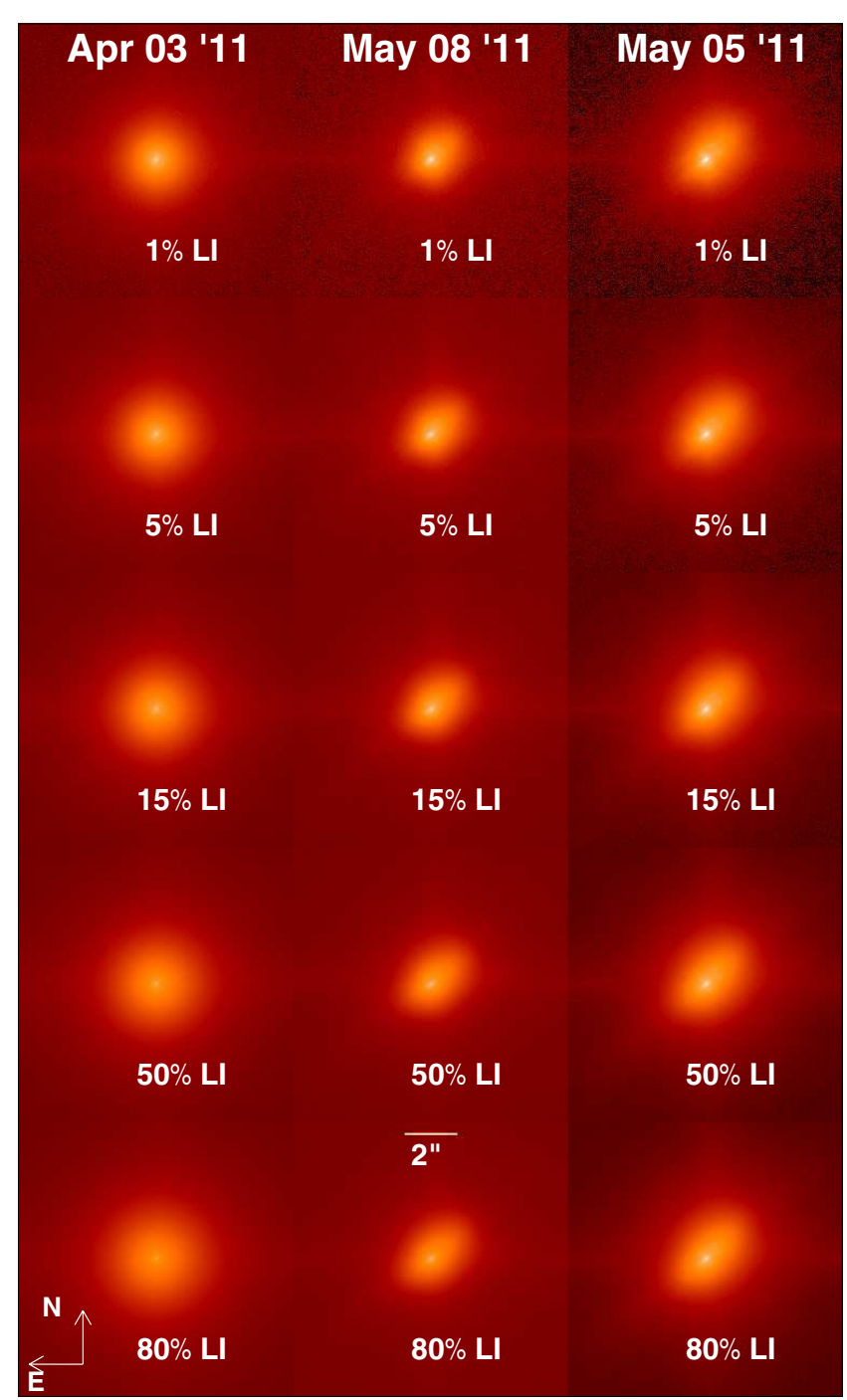

Figure 7. Composite image showing the results of different LI thresholding on the frames acquired with FastCam at the TCS telescope on 2011 April 3, 2011 May 5 and 2011 May 8. This set of images (in logarithmic scale) illustrates the gain in angular resolution close to the target location when applying high restrictive LI thresholds but at the cost of lowering the contrast achieved at large angular distances from target location (see also Figure 8).

(A color version of this figure is available in the online journal.)

No stellar tertiary to HIP 67526 is detected above the "best LI curves."

\subsubsection{Keck Adaptive Optics Imaging}

To further assess the multiplicity of HIP 67526, we acquired high angular resolution images of the star on UT 201224 June using NIRC2 (instrument PI: K. Matthew) with the Keck II AO system (Wizinowich et al. 2000). AO observations probe the immediate vicinity of host stars and generate deep contrast compared to LI (e.g., Fleming et al. 2012; Ma et al. 2013). Furthermore, AO observations are sensitive to objects with red colors given the nominal $1-3 \mu \mathrm{m}$ wavelength operating range.

Our observations consist of dithered frames taken with the $K^{\prime}\left(\lambda_{c}=2.12 \mu \mathrm{m}\right)$ filter. We used the narrow camera setting to provide fine spatial sampling of the NIRC2 PSF. The total on-source integration time was $190 \mathrm{~s}$. Images were processed using standard techniques to replace hot pixel values, flat field the detector array, subtract thermal background noise, and align and coadd frames. 


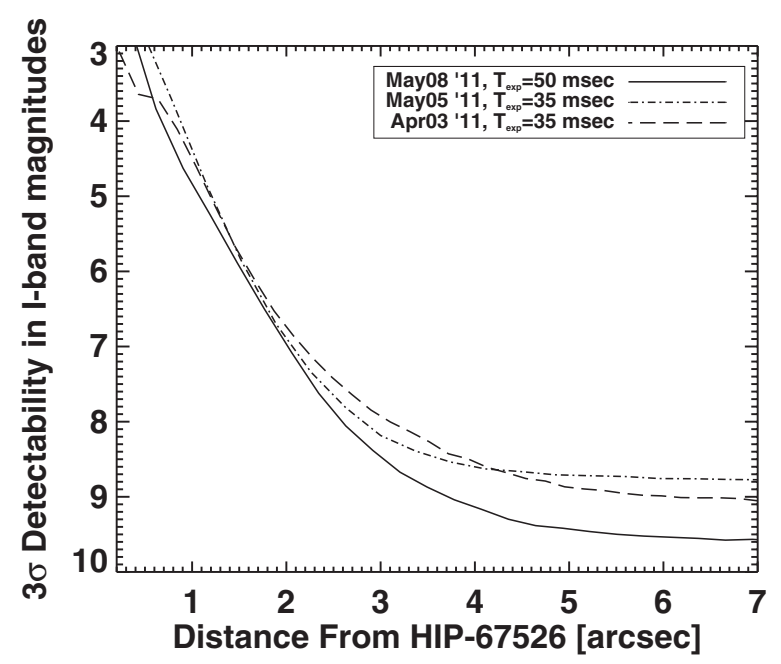

Figure 8. Comparison of the best LI curves achieved on 2011 April 3,2011 May 5, and 2011 May 8 . The $3 \sigma$ detectability $\left(\Delta m_{I}\right)$ curves for individual nights were first computed on the images obtained at different LI thresholds. The best LI curves are the envelope of all detectability curves computed in steps of $1 \%$ of LI thresholding.

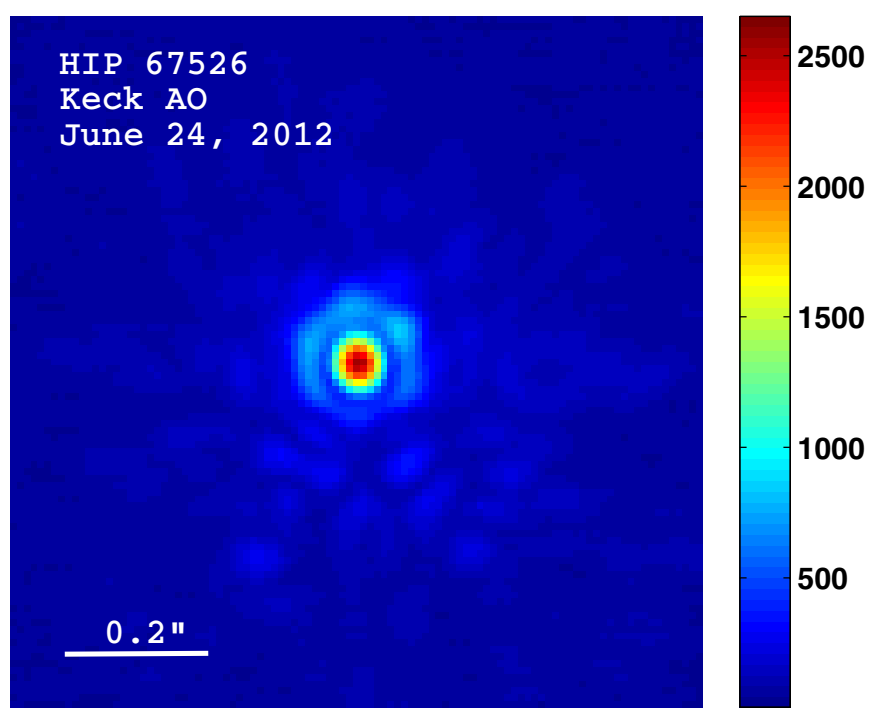

Figure 9. Keck AO image of HIP 67526. No stellar companions are detected with $\Delta m_{K}<5 \mathrm{mag}$ for separations beyond $0^{\prime \prime} .25$ and $\Delta m_{K}<8$ mag for separations beyond $1^{\prime \prime} .0$ at $10 \sigma$ significance level (see also Figure 10).

(A color version of this figure is available in the online journal.)

Figures 9 and 10 show the final reduced AO image and corresponding contrast curve. No candidate companions were noticed in individual raw frames or the final reduced image. Our diffraction-limited observations rule out the presence of companions with $\Delta m_{K}<5$ mag for separations beyond 0 '.25 and $\Delta m_{K}<8 \mathrm{mag}$ for separations beyond 1".0 (10 $\left.\sigma\right)$. We employ the empirical mass-luminosity relationships in Delfosse et al. (2000) to derive the upper mass limit of the undetected companions; this analysis results in an upper mass limit $0.2 M_{\odot}$ for separations larger than $40 \mathrm{AU}$ and $0.1 M_{\odot}$ for separations larger than $100 \mathrm{AU}$.

\section{DISCUSSION AND SUMMARY}

The frequency of BD companions to solar-like stars at close and intermediate separations is less than 1\% (Marcy \& Butler 2000), which is much less than the frequency of planetary

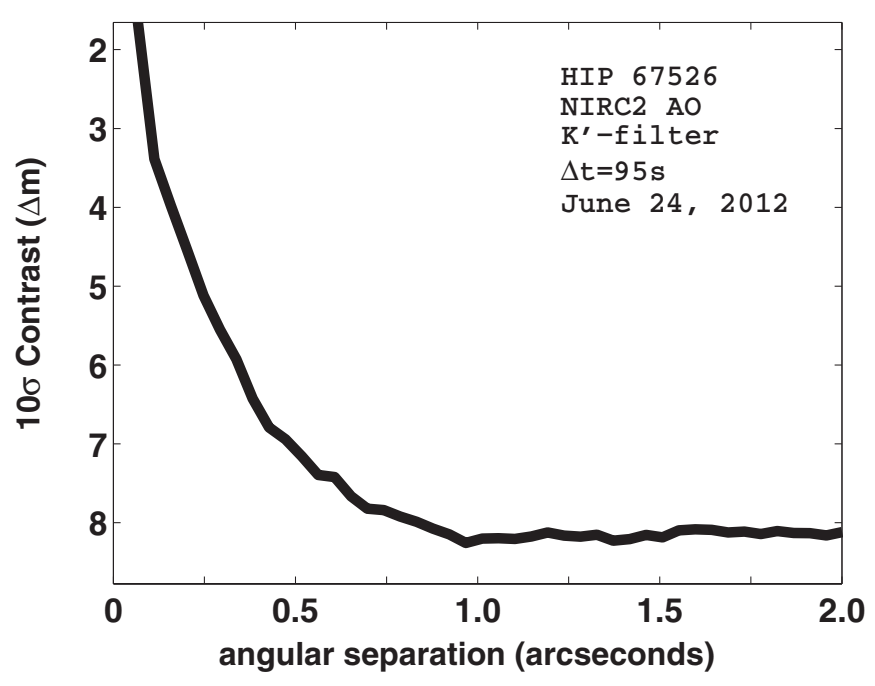

Figure 10. Detectability (contrast curve) for the Keck AO image of HIP 67526.

companions (>10\%; e.g., Howard et al. 2010; Mayor et al. 2011) and the frequency of spectroscopic stellar binaries detected in RV surveys ( 14\%; e.g., Halbwachs et al. 2003). The frequency of BD companions was recently updated by Sahlmann et al. (2011) to be $<0.6 \%$ on the basis of the CORALIE planet-search sample. This result is more accurate since the authors ruled out companions having true masses in the stellar regime using the Hipparcos astrometric measurements to determine the orbital inclinations. Constraining the mass distribution of companions can provide an important observational clue to distinguish the formation and evolution mechanism of planetary, BD, and stellar companions. The current mass distribution suggests that low-mass BD companions less than $\sim 30 M_{\text {Jup }}$ are likely to form in protoplanetary disks, while companions more massive than $\sim 45 M_{\text {Jup }}$ form via fragmentation (Grether \& Lineweaver 2006; Sahlmann et al. 2011; Ma \& Ge 2013). The BD and low-mass stellar companion discoveries from MARVELS will result in a more precise determination of the mass limits of core accretion and gravitational collapse. MARVELS-5b contributes to constraining the shape of the massive BD-low-mass star boundary.

Spectroscopic binaries generally show moderately eccentric orbits (e.g., Duquennoy \& Mayor 1991; Raghavan et al. 2010). Ribas \& Miralda-Escudé (2007) reported a tentative trend that low-mass planets $\left(M \sin i<4 M_{\text {Jup }}\right)$ generally have lower eccentricity than high-mass planets $\left(M \sin i>4 M_{\mathrm{Jup}}\right)$, having a similar eccentricity distribution as binary stars (Figure 3 of Ribas \& Miralda-Escudé 2007). Díaz et al. (2012) reported that most of the BD companions in their sample exhibit a considerable orbital eccentricity, supporting the eccentricity-mass trend. MARVELS-5b has a high eccentricity $(\sim 0.44)$, which is around the peak of the eccentricity distribution of the observed BD and low-mass stellar companions (Sahlmann et al. 2011; Díaz et al. 2012). In view of MARVELS-5b's eccentricity, it is probably a member of the main population of these massive companions to solar-like stars. Our previous MARVELS discoveries (MARVELS-2,3,4,6b) all have an eccentricity lower than $\sim 0.2$ (De Lee et al. 2013).

A stellar tertiary is likely to affect the formation and evolution of the substellar companion to the primary. Observationally, Zucker \& Mazeh (2002) point out that planets found in binaries may have a negative period-mass correlation rather than the positive correlation between the masses and periods of the 
planets orbiting single stars. By studying a larger sample (19 planets in a double or multiple star system), Eggenberger et al. (2004, 2007) showed that short-period $(P<40$ days) planets found in multiple star systems may follow a different periodeccentricity distribution than the short-period planets around isolated stars. These observations seem to indicate that the presence of a stellar companion alters the migration and mass growth rates of planets (Kley 2001). Similar influences have also been observed on close spectroscopic binaries in triple systems. Shorter period binaries are more likely to be in multiple-star systems, i.e., $\sim 80 \%$ for $P<7$ days versus $\sim 40 \%$ for $P>7$ days (Tokovinin et al. 2006). This significant difference suggests that the periods of close binary systems with triples were efficiently decreased by angular momentum exchange with companions.

With masses between planetary companions and stellar components in spectroscopic binaries, the formation and migration of BD and low-mass stellar companions can certainly be affected by the presence of a tertiary as well. However, this problem has not been studied in a statistical way since the current BD and low-mass stellar companion sample is fairly small and no systematic survey of stellar tertiaries for these companions has been conducted. Using high-contrast imaging, the MARVELS survey goes to great lengths to investigate the statistics of its own discoveries of low-mass companions in the presence/absence of a stellar tertiary. As mentioned in Section 1, most of the previous MARVELS discoveries have a stellar tertiary (or a candidate stellar tertiary) detected either by high-contrast imaging or analysis of the long-term RV trend. Among the confirmed discoveries, MARVELS-3b (Wisniewski et al. 2012) has an orbital period $(P \sim 79$ days $)$ and minimum mass ratio $\left(q_{\text {min }} \sim 0.09\right)$ similar to MARVELS-5b (this work), but the former has a less eccentric orbit ( $e \sim 0.1)$. Wisniewski et al. (2012) found a faint candidate tertiary companion on the Keck AO image, separated by $\sim 1^{\prime \prime}$ from the primary, thus speculating that MARVELS-3b might have initially formed in a tertiary system with much different orbital parameters and reached its current short-period orbit during the cluster dispersal phase. For MARVELS-5b, the Keck AO imaging rules out any star with mass greater than $0.1 M_{\odot}$ at a separation larger than $1^{\prime \prime}$ from the primary. This may imply that other formation mechanisms of low-mass-ratio binaries are needed.

In summary, we report a candidate BD or low-mass stellar companion to the solar-like star HIP 67526. The best Keplerian orbital fit parameters were found to have an orbital period of $90.2695_{-0.0187}^{+0.0188}$ days, an eccentricity of $0.4375 \pm 0.0040$, and a semi-amplitude of $2948.14_{-16.55}^{+16.65} \mathrm{~m} \mathrm{~s}^{-1}$. The minimum companion mass was determined to be $65.0 \pm 2.9 M_{\mathrm{Jup}}$. This object helps to populate the high-mass end of the sparsely populated region of the mass function of companions to solartype stars and provide observational evidence to constrain formation and evolution theories. No stellar tertiary is detected with high-contrast imaging for the MARVELS-5 system, while all the other previous MARVELS-discovered systems appear to have at least one stellar companion.

Funding for the MARVELS multi-object Doppler instrument was provided by the W. M. Keck Foundation and NSF with grant AST-0705139. The MARVELS survey was partially funded by the SDSS-III consortium, NSF grant AST-0705139, NASA with grant NNX07AP14G and the University of Florida. Funding for SDSS-III has been provided by the Alfred P. Sloan Foundation, the Participating Institutions, the National Science Foundation, and the U.S. Department of Energy. The SDSS-III Web site is http://www.sdss3.org/. SDSS-III is managed by the Astrophysical Research Consortium for the Participating Institutions of the SDSS-III Collaboration including the University of Arizona, the Brazilian Participation Group, University of Cambridge, University of Florida, the French Participation Group, the German Participation Group, the Michigan State/Notre Dame/JINA Participation Group, Johns Hopkins University, Lawrence Berkeley National Laboratory, Max Planck Institute for Astrophysics, New Mexico State University, New York University, the Ohio State University, University of Portsmouth, Princeton University, University of Tokyo, the University of Utah, Vanderbilt University, University of Virginia, University of Washington, and Yale University.

This work has made use of observations taken with the Telescopio Nationale Galileo (TNG) operated on the island of La Palma by the Foundation Galileo Galilei, funded by the Instituto Nazionale di Astrofisica (INAF), in the Spanish Observatorio del Roque de los Muchachos of the Instituto de Astrofísica de Canarias (IAC).

We have used data from the WASP public archive in this research. The WASP consortium comprises of the University of Cambridge, Keele University, University of Leicester, The Open University, The Queen's University Belfast, St. Andrews University, and the Isaac Newton Group. Funding for WASP comes from the consortium universities and from the UK's Science and Technology Facilities Council. The publication makes use of data products from the Two Micron All Sky Survey, which is a joint project of the University of Massachusetts and the Infrared Processing and Analysis Center/California Institute of Technology, funded by the National Aeronautics and Space Administration and the National Science Foundation.

P.J. acknowledges supports from the Natural Science Foundation of China with grants NSFC 11233002, NSFC 11203022 and the Fundamental Research Funds for the Central Universities. This research is partially supported by funding from the Center for Exoplanets and Habitable Worlds. The Center for Exoplanets and Habitable Worlds is supported by the Pennsylvania State University, the Eberly College of Science, and the Pennsylvania Space Grant Consortium. Keivan Stassun, Leslie Hebb, and Joshua Pepper acknowledge funding support from the Vanderbilt Initiative in Data-Intensive Astrophysics (VIDA) from Vanderbilt University, and from NSF Career award AST-0349075. E.A. thanks NSF for CAREER grant 0645416. G.F.P.M. acknowledges financial support from $\mathrm{CNPq}$ grant No. 476909/2006-6 and FAPERJ grant No. APQ1/26/ 170.687/2004. L.G. acknowledges financial support provided by the PAPDRJ CAPES/FAPERJ Fellowship. J.P.W. acknowledges support from NSF Astronomy and Astrophysics Postdoctoral Fellowship AST 08-02230. L.D.F. acknowledges financial support from CAPES. Work by B.S.G. was supported by NSF CAREER Grant AST-1056524.

\section{REFERENCES}

Baluev, R. V. 2008, MNRAS, 389, 1375

Baraffe, I., Chabrier, G., Barman, T. S., Allard, F., \& Hauschildt, P. H. 2003, A\&A, 402, 701

Basri, G. 2000, ARA\&A, 38, 485

Bouchy, F., Bonomo, A. S., Santerne, A., et al. 2011, A\&A, 533, A83

Burgasser, A. J., Reid, I. N., Siegler, N., et al. 2007, in Protostars and Planets V, ed. B. Reipurth, D. Jewitt, \& K. Keil (Tucson, AZ: Univ. Arizona Press), 427

Burrows, A., Marley, M., Hubbard, W. B., et al. 1997, ApJ, 491, 856 Butler, R. P., Marcy, G. W., Williams, E., et al. 1996, PASP, 108, 500 Butters, O. W., West, R. G., Anderson, D. R., et al. 2010, A\&A, 520, L10 
Castelli, F., \& Kurucz, R. L. 2004, arXiv:astro-ph/0405087

Chabrier, G., Baraffe, I., Allard, F., \& Hauschildt, P. 2000, ApJL, 542, L119 Cumming, A. 2004, MNRAS, 354, 1165

De Lee, N., Ge, J., Crepp, J. R., et al. 2013, AJ, 145, 155

Delfosse, X., Forveille, T., Ségransan, D., et al. 2000, A\&A, 364, 217

Demarque, P., Woo, J.-H., Kim, Y.-C., \& Yi, S. K. 2004, ApJS, 155, 667

Díaz, R. F., Santerne, A., Sahlmann, J., et al. 2012, A\&A, 538, A113

Duquennoy, A., \& Mayor, M. 1991, A\&A, 248, 485

Eggenberger, A., Udry, S., Chauvin, G., et al. 2007, A\&A, 474, 273

Eggenberger, A., Udry, S., \& Mayor, M. 2004, A\&A, 417, 353

Eisenstein, D. J., Weinberg, D. H., Agol, E., et al. 2011, AJ, 142, 72

Erskine, D. J. 2002, PASP, 115, 255

Erskine, D. J., Edelstein, J., Feuerstein, W. M., \& Welsh, B. 2003, ApJ, 592, 103

Femenía, B., Rebolo, R., Pérez-Prieto, J. A., et al. 2011, MNRAS, 413, 1524

Fleming, S. W., Ge, J., Barnes, R., et al. 2012, AJ, 144, 72

Fleming, S. W., Ge, J., Mahadevan, S., et al. 2010, ApJ, 718, 1186

Ford, E. B. 2006, ApJ, 642, 505

Ford, E. B., \& Gregory, P. C. 2007, in ASP Conf. Ser. 371, Statistical Challenges in Modern Astronomy IV, ed. G. Jogesh Babu \& Eric D. Feigelson (San Francisco, CA: ASP), 189

Ge, J. 2002, ApJL, 571, L165

Ge, J., \& Eisenstein, D. 2009, in Astro2010: The Astronomy and Astrophysics Decadal Survey, Science White Papers, 2010, 86

Ge, J., Erskine, D. J., \& Rushford, M. 2002, PASP, 114, 1016

Ge, J., Lee, B., de Lee, N., et al. 2009, Proc. SPIE, 7440, 18

Ge, J., Mahadevan, S., Lee, B., et al. 2008, in ASP Conf. Ser. 398, Extreme Solar Systems, ed. D. Fischer, F. A. Rasio, S. E. Thorsett, \& A. Wolszczan (San Francisco, CA: ASP), 449

Ge, J., van Eyken, J., Mahadevan, S., et al. 2006, ApJ, 648, 683

Gelman, A., Carlin, J. B., Stern, H. S., \& Rubin, D. B. 2003, Bayesian Data Analysis (New York: Chapman and Hall)

Gelman, A., \& Rubin, D. B. 1992, StaSc, 7, 457

Gratton, R. G., Bonanno, G., Bruno, P., et al. 2001, ExA, 12, 107

Gregory, P. C. 2005, ApJ, 631, 1198

Grether, D., \& Lineweaver, C. H. 2006, ApJ, 640, 1051

Gunn, J. E., Siegmund, W. A., Mannery, E. J., et al. 2006, AJ, 131, 2332

Halbwachs, J. L., Mayor, M., Udry, S., \& Arenou, F. 2003, A\&A, 397,159

Hauschildt, P. H., Allard, F., \& Baron, E. 1999, ApJ, 512, 377

Howard, A. W., Marcy, G. W., Johnson, J. A., et al. 2010, Sci, 330, 653

Johnson, J. A., Aller, K. M., Howard, A. W., \& Crepp, J. R. 2010, PASP, 122, 905

Kharchenko, N. V., \& Roeser, S. 2009, yCat, 1280, 0

Kirkpatrick, J. D., Reid, I. N., Liebert, J., et al. 1999, ApJ, 519, 802

Kirkpatrick, J. D., Reid, I. N., Liebert, J., et al. 2000, AJ, 120, 447

Kirkpatrick, J. D., Cushing, M. C., Gelino, C. R., et al. 2011, ApJS, 197,19

Kley, W. 2001, in IAU Symp. 200, The Formation of Binary Stars, ed. Hans Zinnecker \& Robert D. Mathieu (Cambridge: Cambridge Univ. Press), 511
Kurucz, R. 1993, ATLAS9 Stellar Atmosphere Programs and 2 km s1 Grid, Kurucz CD-ROM No. 13 (Cambridge, MA: SAO), 13

Latham, D. W., Stefanik, R. P., Mazeh, T., Mayor, M., \& Burki, G. 1989, Natur, 339,38

Lee, B. L., Ge, J., Fleming, S. W., et al. 2011, ApJ, 728, 32

Lomb, N. R. 1976, Ap\&SS, 39, 447

Ma, B., \& Ge, J. 2013, arXiv:1303.6442

Ma, B., Ge, J., Barnes, R., et al. 2013, AJ, 145, 20

Mack, C. E., III, Ge, J., Deshpande, R., et al. 2013, AJ, 145, 139

Mamajek, E. E., \& Hillenbrand, L. A. 2008, ApJ, 687, 1264

Marcy, G. W., \& Butler, R. P. 1996, ApJL, 464, L147

Marcy, G. W., \& Butler, R. P. 2000, PASP, 112, 137

Masana, E., Jordi, C., \& Ribas, I. 2006, A\&A, 450, 735

Mayor, M., \& Queloz, D. 1995, Natur, 378, 355

Mayor, M., \& Udry, S. 2000, in ASP Conf. Ser. 219, Disks, Planetesimals, and Planets, ed. F. Garzón, C. Eiroa, D. de Winter, \& T. J. Mahoney (San Francisco, CA: ASP), 441

Mayor, M., et al. 2011, arXiv:1109.2497

Oppenheimer, B. R., Kulkarni, S. R., Matthews, K., \& Nakajima, T. 1995, Sci, 270,1478

Oscoz, A., et al. 2008, Proc. SPIE, 7014, 701447

Pont, F., Melo, C. H. F., Bouchy, F., et al. 2005, A\&A, 433, L21

Pourbaix, D. 2001, A\&A, 369, L22

Raghavan, D., McAlister, H. A., Henry, T. J., et al. 2010, ApJS, 190, 1

Rebolo, R., Zapatero Osorio, M. R., \& Martín, E. L. 1995, Natur, 377, 129

Ribas, I., \& Miralda-Escudé, J. 2007, A\&A, 464, 779

Ruiz, M. T., Leggett, S. K., \& Allard, F. 1997, ApJL, 491, L107

Sahlmann, J., Ségransan, D., Queloz, D., et al. 2011, A\&A, 525, A95

Santos, N. C., Israelian, G., \& Mayor, M. 2001, A\&A, 373, 1019

Scargle, J. D. 1982, ApJ, 263, 835

Spiegel, D. S., Burrows, A., \& Milsom, J. A. 2011, ApJ, 727, 57

Tinney, C. G., Delfosse, X., \& Forveille, T. 1997, ApJL, 490, L95

Tokovinin, A., Thomas, S., Sterzik, M., \& Udry, S. 2006, A\&A, 450, 681

Torres, G., Andersen, J., \& Giménez, A. 2010, A\&ARv, 18, 67

Valenti, J. A., \& Fischer, D. A. 2005, ApJS, 159, 141

van Eyken, J. C., Ge, J., \& Mahadevan, S. 2011, ApJS, 189, 156

van Leeuwen, F. (ed.) 2007, Hipparcos, the New Reduction of the Raw Data (Astrophysics and Space Science Library, Vol. 350; Berlin: Springer)

Vogt, S. S., Butler, R. P., Marcy, G. W., et al. 2002, ApJ, 568, 352

Wang, J., \& Ford, E. B. 2011, MNRAS, 418, 1822

Wang, J., Ge, J., Jiang, P., \& Zhao, B. 2011, ApJ, 738, 132

Wang, J., Ge, J., Wan, X., De Lee, N., \& Lee, B. 2012a, PASP, 124, 1159

Wang, J., Ge, J., Wan, X., Lee, B., \& De Lee, N. 2012b, PASP, 124, 598

Wang, S.-I., Hildebrand, R. H., Hobbs, L. M., et al. 2003, Proc. SPIE, 4841,1145

Wisniewski, J. P., Ge, J., Crepp, J. R., et al. 2012, AJ, 143, 107

Wizinowich, P., Acton, D. S., Shelton, C., et al. 2000, PASP, 112, 315

Wright, J. T. 2005, PASP, 117, 657

Wright, J. T., Roy, A., Mahadevan, S., et al. 2013, ApJ, 770, 119

Zucker, S., \& Mazeh, T. 2002, ApJL, 568, L113 